REVIEW ARTICLE

\title{
Adenosine Deaminase in Diagnosis of Tuberculosis: A Review
}

\author{
*R Barua1, MA Hossain ${ }^{2}$ \\ ${ }^{1}$ Assistant Professor, Department of Microbiology, Shaheed Suhrawardy Medical College \\ Sher-e-bangla Nagar, Dhaka \\ ${ }^{2} J u n i o r$ Consultant (Medicine), Bangabandhu Sheikh Mujib Medical University, Dhaka
}

*Corresponding Author

\section{ABSTRACT}

The diagnosis of tuberculosis (TB) continues to be a challenge in clinical practice. Traditional diagnostic methods are very useful but do not provide enough sensitivity and specificity. Adenosine deaminase (ADA) has been developed and widely used for the diagnosis of TB. ADA is an enzyme that increases in TB because of the stimulation of T-cell lymphocytes by mycobacterial antigens. This article reviews the characteristics, metabolism and clinical uses of ADA for the diagnosis of TB in clinical practices. There is sufficient data supporting yield of ADA in various body fluids for the diagnosis of TB. ADA may be used for early diagnosis of $\mathrm{TB}$, especially in case of negative acid fast bacilli (AFB) smear from the body specimens.

Keywords: Adenosine deaminase, ADA, tuberculosis, diagnosis

\section{Introduction}

Tuberculosis (TB) is a bacterial disease caused by the tubercle bacilli which includes Mycobacterium tuberculosis. TB remains one of the major health problems in Bangladesh and worldwide. Globally 8.6 million people developed TB and 1.3 million died from the disease (including 320000 deaths among HIVpositive people). Bangladesh ranks sixth among the world's 22 high-burden TB countries with estimated 350,000 new cases and 70,000 deaths per year ${ }^{1}$. The lung tissue is involved in pulmonary $\mathrm{TB}$ and the tissue other than lung tissue like pleural fluid, ascitic fluid etc is involved in extrapulmonary TB (EPTB). Prompt diagnosis is essential for effective TB control programme. Although we have many methods for the diagnosis of pulmonary $\mathrm{TB}$, for example Ziehl-Neelsen (Z-N) staining, polymerase chain reaction (PCR) and culture, these methods do not provide enough sensitivity and specificity. The sensitivities of $\mathrm{ZN}$ staining and culture are $10-40 \%$ and $8-49 \%$ respectively in the diagnosis of TB infection ${ }^{2}$. The definitive diagnosis of EPTB depends on the demonstration of Mycobacterium tuberculosis in the specimens like pleural fluid, ascitic fluid, pericardial fluid, cerebrospinal fluid (CSF) or pleural biopsy specimen, and can also be established with reasonable certainty by demonstration of granuloma in the parietal pleura, peritoneum, pericardium etc ${ }^{3}$. Although mycobacterial culture is the gold standard in diagnosing TB, Mycobacterium spp. grows very slowly and it can take up to six weeks to isolate it in culture. Determination of susceptibility to drugs can add another three to six weeks to the process. Meanwhile the disease may progress and be transmitted to others when appropriate treatment is delayed. There is a need of a simple, rapid and reliable test which can be easily carried out in the clinical laboratory. Thoracoscopy in diagnosing tubercular serositis offers a near $100 \%$ positive diagnostic yield on histology and $76 \%$ positive on culture ${ }^{3,4}$. However, historically, since pleural biopsy is more invasive and hazardous than thoracocentesis, alternative diagnostic approaches have been extensively evaluated ${ }^{5}$. Adenosine deaminase (ADA) has been developed and widely used for the diagnosis of TB due to its simplicity, low cost, and quickly available results. Many studies have confirmed the high sensitivity and 
specificity of ADA (sensitivity $92 \%$ and specificity $89 \%$ ) for early diagnosis of EPTB, such as tuberculous pleuritis, pericarditis, ascites and meningitis ${ }^{6}$. The author aimed to review the characteristics, its metabolism and clinical uses of ADA for the diagnosis of TB.

\section{Characteristics, metabolism and assay of adenosine deaminase (ADA)}

ADA is an enzyme catalyzing the deamination reaction from adenosine to inosine. It is also an essential enzyme of the purine catabolic pathway. There are 2 isoforms of ADA, ADA- 1 and ADA2. ADA-1 is found in many tissues including red blood cells. ADA-2 is found only in macrophages and monocytes ${ }^{7,8}$. ADA acts in proliferation and differentiation of lymphocyte, especially $\mathrm{T}$ lymphocyte. It also acts in maturation of monocytes transforming them to macrophage. ADA is a significant indicator of active cellular immunity. It increases in biological fluids in the course of infectious disease characterized by micro-organisms infecting the macrophages. For example, deficiency in ADA in humans manifests primarily as severe lymphopenia and immunodeficiency ${ }^{9}$. Furthermore, ADA has been proposed to be a useful surrogate marker for TB because it can be detected in body fluids such as pleural, pericardial and peritoneal fluid. The levels of ADA increase in TB because of the stimulation of $\mathrm{T}$ cells by mycobacterial antigens ${ }^{8}$.

\section{Adenosine deaminase (ADA) activity assay}

Total plasma ADA can be measured by a spectrophotometric method described by Guisti and Galanti in 1984. It uses the principles that the ADA assay is based on the enzymatic deamination of adenosine to inosine and formation of ammonia. Ammonia $\left(\mathrm{NH}_{3}\right)$ forms under conversion of adenosine causing an intensely blue indophenol with sodium hypochlorite and phenol in an alkaline solution as determined by modification of a Berthelot's reaction. Sodium nitroprusside is used as the catalyst. The ammonia concentration is directly proportional to the absorbance of the indophenols measured at a wavelength of $620 \mathrm{~nm}$. The reaction catalyzed by $\mathrm{ADA}$ is stopped at the end of one hour incubation at $37^{\circ} \mathrm{C}$ by the addition of phenol nitroprusside solution. ADA activity is expressed in international units (IU) using the formula as follows: (Absorbance of sample/ Absorbance of standard) x $50 \mathrm{IU} / 1^{10}$.

Comparison of the performance of adenosine deaminase assay using Diazyme ${ }^{\mathrm{R}}$ commercial kit and Giusti including modified Giusti method

The determination of ADA levels has been performed using the method proposed by Giusti which has undergone certain modifications over time $^{10}$. The modified Giusti method including the Berthelot reaction is developed in order to obtain better results. But there is a lack of standardization and it does not allow this assay to be used as a good diagnostic test ${ }^{11}$. An automated method (Diazyme ${ }^{\mathrm{R}}$ commercial kit, Diazyme Laboratories, San Diego, CA, USA) with automation (ADVIA 1650 analyzer; Bayer Diagnostics, Tarrytown, NY, USA) was developed for the determination of ADA activity in pleural fluid and CSF samples. A study had compared the results with those obtained from the modified Giusti method, which is considered a reference test for biochemical study ${ }^{12}$. The values found for the pleural fluid and CSF confirmed the strong correlation between these 2 methods. Pleural fluid with cut-off values of 40 $\mathrm{U} / \mathrm{L}$ (conventional method) and $30 \mathrm{U} / \mathrm{L}$ (automated method), had the concordance of 96.8 percent. For the detection in CSF samples, the cut-off value was $9 \mathrm{U} / \mathrm{L}$ (for both methods) and the concordance was 100 percent. Therefore, the reference values for the diagnosis of $\mathrm{TB}$ in pleural fluid samples are $40 \mathrm{U} / \mathrm{L}$ (modified Giusti method) and $30 \mathrm{U} / \mathrm{L}$ (automated method: Diazyme R commercial kit), versus 9 $\mathrm{U} / \mathrm{L}$ (for both methods) in CSF samples.

Use of adenosine deaminase in clinical practices stratified by body fluids

\section{Pleural effusion}

Almost all research workers have shown sensitivity and specificity of $90 \%$ to $100 \%$ for the value of ADA in pleural fluid using different cut off levels. Gupta et al. showed sensitivity 
and specificity for diagnosing tuberculous pleural effusion of $100 \%$ and $94.1 \%$ respectively ${ }^{13}$, which was almost similar to the study done by Burgess et al. (sensitivity 90\% and specificity $89 \%$ at cut-off value of 50 $\mathrm{U} / \mathrm{L})^{14}$, Strankinga et al. (sensitivity $100 \%$ and specificity $87 \%$ at cut-off value of $53 \mathrm{U} / \mathrm{L})^{15}$ and Farhana et al. (sensitivity $95 \%$ and specificity $83.3 \%$ at cut-off value of $40 \mathrm{U} / \mathrm{L}) 16$.

\section{Bronchoalveolar lavage fluid}

The role of ADA estimation in bronchoalveolar lavage (BAL) fluid for diagnostic of smearnegative pulmonary TB is not clearly useful. A prospective study was conducted in Thailand to determine the diagnostic value of ADA activity in BAL fluid for diagnosis of pulmonary TB among 148 patients with abnormal chest X-ray and unknown definite diagnosis. All patients were either sputum-smear negative for AFB or failed to produce sputum. The mean ADA activity in the BAL for pulmonary $\mathrm{TB}$, malignancy and miscellaneous causes groups was $8.98,7.63$ and $11.61 \mathrm{U} / 1$ respectively. There was no difference of ADA levels in BAL fluid among these 3 groups of patients ${ }^{17}$.

\section{Ascites}

Ascites is the predominant finding and it is present in about $78 \%$ of patients with tuberculous peritonitis ${ }^{18}$. A systematic review from 35 studies of patients with tuberculous peritonitis was conducted and the authors aimed to determine the performance of the available tests for diagnosis of tuberculous peritonitis ${ }^{19}$. Because of low sensitivity of the current methods like ascites total protein, serum-ascites albumin gradient, Ziehl-Neelsen staining and culture $^{20}$, ADA may be a better diagnostics for tuberculous peritonitis. Ascites ADA is increased in tuberculous ascitic fluid because of the stimulation of $\mathrm{T}$ cells by the mycobacterial antigens. ADA activity in the peritoneal fluid has been proved to be a simple and reliable method for early diagnosis of tuberculous peritonitis $^{21}$. Sensitivity and specificity levels over 90 percent have been reported ${ }^{22}$ with the exception of a study by Hillebrand et al. ${ }^{23}$, who reported a sensitivity of $59 \%$. Lower sensitivity may have been related to the higher incidence of cirrhosis in the study group of patients. These observations were countered by Burgess et al. ${ }^{24}$, when they evaluated cirrhotic patients with tuberculous peritonitis and reported a sensitivity of 94 percent. At present, an ascites ADA activity of $30 \mathrm{U} / \mathrm{L}$ is generally accepted as the cut-off level expected to yield the best results. Hortiwakul et al. studied ADA testing among the patients with tuberculous peritonitis. Using ROC curves, a cut-off value of $22 \mathrm{U} / \mathrm{L}$ for the diagnosis was found to yield the best results; corresponding sensitivity and specificity were $84.8 \%$ and $82.6 \%$, respectively 25 . In the study done by Voight et al. the mean ADA level for tubercular etiology was $99.8 \mathrm{U} / \mathrm{L}$ with sensitivity and specificity for diagnosis tubercular ascites was $95 \%$ and $98 \%$ respectively ${ }^{26}$, which was similar to the study done by Dwivedi et al. (sensitivity $100 \%$ and specificity $96.6 \%$ at an ADA level $>33 \mathrm{U} / \mathrm{L})^{27}$ and Gupta et al. (sensitivity $95 \%$ and specificity $94.1 \%$ at an ADA level $>30 \mathrm{U} / \mathrm{L})^{28}$.

\section{Cerebrospinal fluid}

Chotmongkol et al. conducted a study comparing the ADA activity in cerebrospinal fluid (CSF) between patients with tuberculous and non-tuberculous meningitis was conducted. The ROC curve identified a CSF ADA level of $15.5 \mathrm{U} / 1$ as the best cut-off value to differentiate between the 2 groups, with a sensitivity of $75 \%$, specificity of $93 \% 29$. In bacterial meningitis, mean ADA is quite high when compared with non-tuberculous and non-bacterial meningitis group. The yield of ADA may be low in setting to differentiate bacterial from tuberculosis meningitis. The possible explanation may be from ADA value in most assays detected total ADA which includes ADA-1 and ADA-2. Thus, fluid with high cell counts (e.g. bacterial meningitis) can have high total ADA and may be undifferentiated from tuberculous meningitis. ADA activity in the CSF of HIV-infected patients had limited value for diagnosis of tuberculous meningitis. A retrospective study 
was conducted to determined ADA levels in 417 CSF samples from HIV-infected patients with neurological symptoms ${ }^{30}$. HIV-associated neurological disorders and progressive multifocal leukoencephalopathy were not associated with elevated ADA in CSF. When using a cut-off point of $8.5 \mathrm{IU} / 1$ for the diagnosis of tuberculous meningitis, sensitivity was only $57 \%$ and specificity was $87 \%$. A cutoff value of 10 IU/l gave a specificity of $90 \%$ but very low sensitivity $(36 \%)$. The results of this study indicated that ADA determination in CSF has limited utility for the diagnosis of tuberculous meningitis in HIV infected patients. Recommendation from British Infection Society for the diagnosis and treatment of TB of the central nervous system in adults and children suggests that the activity of ADA is raised in the CSF of patients with tuberculous meningitis and has been evaluated as a diagnostic assay ${ }^{31}$. The major problem was lacking of specificity. High CSF ADA activity has been reported from patients with lymphomas, malaria, brucellosis and pyogenic meningitis. Thus, CSF ADA activity is not recommended as a routine diagnostic test for TB of the central nervous system. However, prevalence of tuberculous meningitis in Bangladesh is high and positive predictive value for $\mathrm{ADA}$ in diagnosis of tuberculous meningitis is much higher than that of western countries. The value of CSF ADA may have usefulness in Bangladesh.

\section{Pericardial effusion}

A prospective study in South Africa showed that an ADA cut-off level of $40 \mathrm{U} / 1$ resulted in a test sensitivity, specificity, positive predictive value, negative predictive value and diagnostic efficiency of $84 \%, 80 \%, 91 \%, 66 \%$ and $83 \%$, respectively ${ }^{32}$. Kwan et al. studied pericardial fluid ADA level along with histopathology of pericardial biopsy and found a cut off ADA level of $40 \mathrm{U} / \mathrm{L}$ in pericardial fluid which has sensitivity of $93 \%$ and specificity of $97 \%$ in diagnosis of tubercular pericardial effusion ${ }^{33}$, which is similar to the study by Mathur et al. showed $100 \%$ sensitivity and $83.3 \%$ specificity ${ }^{34}$.

\section{Adenosine deaminase (ADA) versus polymerase chain reaction (PCR)}

Nucleic acid identification by PCR is a rapid, sensitive and specific tool for the detection of Mycobacterium tuberculosis ${ }^{35}$. It permits direct identification of the $\mathrm{M}$. tuberculosis complex and results are available in a day or two. However, sensitivity depends on a target site. PCR targets such as IS6110 and hsp65 kDa yield a sensitivity of $42-100 \%$ and a specificity of $85-100 \% 36$. Sensitivity of PCR was achieved when devR and IS6110 test results were combined; the sensitivity and specificity values were $83 \%$ and $94 \%$ respectively in pleural fluid $^{37}$. A cross-sectional study was performed in a total of 179 body fluid samples. All specimens were analyzed for AFB smear, ADA activity (by a method based on the Berthlot reaction) and multiplex PCR using amplicons such as IS6110, dnaJ gene and hsp65 genes. On comparing AFB and ADA results with PCR, the PCR is clearly more effective than AFB smear $(p<0.001)$ and ADA estimation $(p<0.02)$ in all types of body fluids ${ }^{36}$. Another study collected samples of 67 consecutive patients with large pericardial effusions. Sensitivity and specificity with a cut-off value of $40 \mathrm{U} / \mathrm{L}$ for diagnosis of tuberculous pericarditis were $83 \%$ and $78 \%$, respectively, compared to PCR which sensitivity and specificity were $75 \%$ and $100 \%$ respectively ${ }^{38}$. In this study, PCR had better specificity and positive predictive value than ADA for diagnosis of tuberculous pericarditis, but the sensitivity was not different from ADA. Disadvantages for PCR are: it needs more resources and sophisticated equipments than ADA, price is higher, needs longer time for test results and not every hospitals can set PCR lab (especially small to medium sizes hospitals).

\section{Conclusion}

ADA is an essential enzyme of the purine catabolic pathway catalyzing the deamination reaction from adenosine to inosine that increases in TB because of the stimulation of T-cell lymphocytes by mycobacterial antigens. There is sufficient data supporting yield of ADA in various 
body fluids for the diagnosis of TB. ADA assays can be performed in many health care centres with limited diagnostic facilities other than mycobacterial culture, PCR etc. In addition, it is cheap and it has good sensitivity. ADA may be used for early diagnosis of TB, especially in case of negative AFB smear from the body specimens. However, culture is still the gold standard and mandatory for the confirmatory diagnosis. Prompt treatment of TB is crucial, especially in Bangladesh, where it is a high burden TB area. Further research regarding ADA is necessary to improve specificity, minimize false positive and choose the suitable cut-off value.

\section{References}

1. WHO. Global tuberculosis report 2013. Tuberculosis - epidemiology. WHO Press, World Health Organization, 20 Avenue Appia, 1211 Geneva 27, Switzerland. 2013: 1-11.

2. Jay SJ. Diagnostic procedures for pleural diseases. Symposium on pleural diseases. Clin Chest Med 1985; 6: 33-48.

3. Porcel JM. Tuberculous pleural effusion. Lung 2009; 187: 263-70.

4. Light RW. Update on tuberculous pleural effusion. Respirology 2010; 15: 451-8.

5. Udwadia ZF, Sen T. Pleural tuberculosis: an update. Curr Opin Pulm Med 2010; 16: 399-406.

6. Greco S, Girardi E, Masciangelo R et al Adenosine Deaminase and interferon gamma measurements for the diagnosis of tuberculous pleurisy: a metaanalysis. Int J Tuberc Lung Dis 2003; 7 (8): 777-86.

7. Kaya S, Cetin ES, Aridogan $\mathrm{BC}$ et al Adenosine deaminase activity in serum of patients with hepatitis - a useful tool in monitoring clinical status. J Microbiol Immunol Infect 2007; 40: 288-92.

8. Gakis C. Adenosine deaminase (ADA) isoenzymes ADA1 and ADA2: diagnostic and biological role. Eur Respir J 1996; 9: 632-3.

9. Cimen F, Ciftci TU, Berktas BM et al The relationship between serum adenosine deaminase levels in lung tuberculosis along with drug resistance and the category of tuberculosis. Turkish Respir J 2008; 9: 20-3.
10. Guisti G, Galanti B. Colorimetric method. In: Bergmeyer HU, ed. Methods of enzymatic analysis. Weinheim: Verlag Chemie, 1984: 315-23.

11. Passing H, Bablok. A new biometrical procedure for testing the equality of measurements from two different analytical methods. Application of linear regression procedures for method comparison studies in clinical chemistry, Part I. J Clin Chem Clin Biochem 1983; 21: 709-20.

12. Feres MC, Martino MC, Maldijian $S$ et al Laboratorial validation of an automated assay for the determination of adenosine deaminase activity in pleural fluid and cerebrospinal fluid. J Bras Pneumol 2008; 34: 1033-9.

13. Gupta DK, Suri JC, Goel A. Efficacy of ADA in diagnosis of pleural effusions. 1nd J Chest Dis 1990; 32 (4): 205-8.

14. Burgess LJ. Use of adenosine deaminase as a diagnostic tool for tuberculous pleurisy. Thorax 1995; 50 (6): 672-4.

15. Strankinga WF. Adenosine deaminase activity in tuberculous pleural effusions: a diagnostic test. Tubercle 1987; 68 (2): 137-40.

16. Farhana A, Islam MS, Rehena $\mathrm{Z}$ et al. Adenosine Deaminase and Other Conventional Diagnostic Parameters in Diagnosis of Tuberculous Pleural Effusion. Dinajpur Med Col J 2013; 6 (2): 105-12.

17. Reechaipichitkul W, Lulitanond V, Patjanasoontorn $B$ et al A. Diagnostic yield of adenosine deaminase in bronchoalveolar lavage. Southeast Asian J Trop Med Public Health 2004; 35: 730-4.

18. Sanai FM, Bzeizi KI. Systematic review: tuberculous peritonitis--presenting features, diagnostic strategies and treatment. Aliment Pharmacol Ther 2005; 22: 685-700.

19. Runyon BA, Montano AA, Akriviadis EA et al The serum-ascites albumin gradient is superior to the exudate-transudate concept in the differential diagnosis of ascites. Ann Intern Med 1992; 117: 215-20.

20. Chow KM, Chow VC, Hung LC et al Tuberculous peritonitis-associated mortality is high among patients waiting for the results of mycobacterial cultures of ascitic fluid samples. Clin Infect Dis 2002; 35: 409-13. 
21. Martinez-Vazquez JM, Ocana I, Ribera E et al Adenosine deaminase activity in the diagnosis of tuberculous peritonitis. Gut 1986; 27: 1049-53.

22. Sathar MA, Simjee AE, Coovadia YM, et al. Ascitic fluid gamma interferon concentrations and adenosine deaminase activity in tuberculous peritonitis. Gut 1995; 36: 419-21.

23. Hillebrand DJ, Runyon BA, Yasmineh WG et al Ascitic fluid adenosine deaminase insensitivity in detecting tuberculous peritonitis in the United States. Hepatology 1996; 24: 1408-12.

24. Burgess LJ, Swanepoel CG, Taljaard JJ. The use of adenosine deaminase as a diagnostic tool for peritoneal tuberculosis. Tuberculosis (Edinb) 2001; 81: 243-8.

25. Hortiwakul R, Keeratichananont S, Chayakul P. The use of adenosine deaminase activity (ADA) for the diagnosis of tuberculous peritonitis. Songkla Med J 2005; 23: 87-92.

26. Voight MD, Kalvaria L, Trey C, et al. Diagnostic value of ascites adenosine deaminase activity in tuberculous peritonitis. Lancet 1989; 1: 751-4.

27. Dwivedi M, Misra SP, Misra V, et al. Value of adenosine deaminase estimation in the diagnosis of tuberculous ascites. Am J Gastroenterology 1990; 85: 1123-5.

28. Gupta VK, Mukerji S, Datta SK. Diagnostic evaluation of ADA in tuberculous peritonitis. JAPI 1992; 40 (6): 387-9.

29. Chotmongkol V, Teerajetgul Y, Yodwut C. Cerebrospinal fluid adenosine deaminase activity for the diagnosis of tuberculous meningitis in adults. Southeast Asian J Trop Med Public Health 2006; 37: 948-52.

30. Corral I, Quereda C, Navas E, et al. Adenosine deaminase activity in cerebrospinal fluid of HIVinfected patients: limited value for diagnosis of tuberculous meningitis. Eur J Clin Microbiol Infect Dis 2004; 23: 471-6.
31. Thwaites G, Fisher M, Hemingway C et al British Infection Society guidelines for the diagnosis and treatment of tuberculosis of the central nervous system in adults and children. J Infect 2009; 59: 167-87.

32. Reuter H, Burgess LJ, Carstens ME et al Adenosine deaminase activity--more than a diagnostic tool in tuberculous pericarditis. Cardiovasc J S Afr 2005; 16: 143-7.

33. Kin JH Adenosine deaminase and CEA in pericardial effusion. Circulation 1994; 89 (6): 272835 .

34. Mathur PC, Tiwari KK, Trikha S et al Diagnostic value of adenosine deaminase (ADA) activity in tubercular serositis. Indian J Tuberc 2006; 53: 92-5.

35. Hawkey PM. The role of the polymerase chain reaction in the diagnosis of mycobacterial infections. Rev Med Microbiol 1994; 4: 21-32.

36. Bandyopadhyay D, Gupta S, Banerjee S, et al. Adenosine deaminase estimation and multiplex polymerase chain reaction in diagnosis of extrapulmonary tuberculosis. Int $\mathbf{J}$ Tuberc Lung Dis 2008; 12: 1203-8.

37. Chakravorty S, Sen MK, Tyagi JS. Diagnosis of extrapulmonary tuberculosis by smear, culture, and PCR using universal sample processing technology. J Clin Microbiol 2005; 43:4357-62.

38. Lee JH, Lee CW, Lee SG, et al. Comparison of polymerase chain reaction with adenosine deaminase activity in pericardial fluid for the diagnosis of tuberculous pericarditis. Am J Med 2002; 113: 51921. 